\title{
TRATAMIENTO COADYUVANTE DE LA ENFERMEDAD PELVICA INFLAMATORIA AGUDA CON INDOMETACINA
}

\author{
Doctores Alvaro Fonnegra Miramón * y Laureano Marín Ardila**
}

Todos conocemos el efecto anti-flogístico y analgésico de a!gunas drogas del grupo químico de la Fenilbutazona en la enfermedad pélvica inflamatoria aguda.

Desde el punto de vista de la acción de esos medicamentos sobre tales entidades, no tenemos nada que desear, pero no es así la opinión sobre los efectos secundarios, los cuales sí tienen mucha importancia, ya que después de cierto tiempo de su administración en dosis terapéuticas dejan mostrar algunas manifestaciones indeseables, por todos conocidas y fundamentalmente más ostensibles en la mujer que en el hombre. Estos efectos secundarios, esencia!mente se localizan en: tracto digestivo, renal, sistema nervioso, aparato cardiovascu-

* Profesor Asociado.

Residente de Cuarto Año.

Sección de Obstetricia y Ginecología. Trabajo presentado al VI Congreso de Obstetricia y Ginecología por la Sección de Obstetricia y Ginecología de la Facultad de Medicina de la Universidad Nacional de Colombia.

Hospital San Juan de Dios, Bogotá, D. $\mathrm{E}$ lar, alteraciones hematológicas. Se debe anotar además la influencia sobre el sistema endocrino, siendo de especial mención el efecto sobre el ciclo menstrual, observándose disminución de intensidad o interrupción de la hemorragia menstrual. (Acción que está en estudio $y$ que no se ha comprobado plenamente).

Teniendo en cuenta estos efectos y otros no mencionados, hemos querido probar un medicamento muy utilizado en procesos inflamatorios articulares, cuyos resultados anti-flogísticos y analgésicos han sido sorprendentes, sin presentar efectos secundarios tan notorios como los ya anotados en relación con la Fenil-Butazona.

Se trata, pues, de la Indometacina, cuya fórmula estructural es la siguiente:

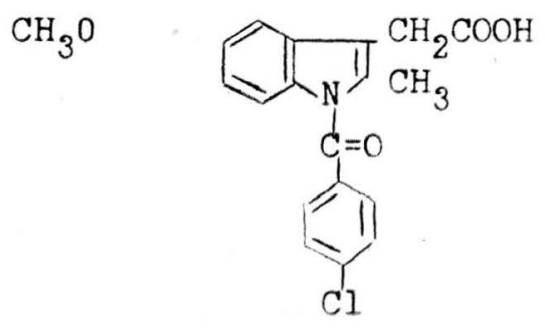


Material y métodos

Para esta experiencia se utilizaron 41 pacientes con enfermedad pélvica inflamatoria aguda discriminados en la siguiente forma:

\section{Cuadro 1}

DISCRIMINACION DE AFECCIONES TRATADAS CON INDOMETACINAPENICILINA

\begin{tabular}{lrr}
\hline & Casos & $\%$ \\
\hline Anexitis agudas & 21 & 51.2 \\
Salpingitis catarral & 6 & 14.6 \\
Abscesos tubo-ováricos & 8 & 19.5 \\
Pelviperitonitis & 5 & 12.2 \\
Ectopico roto e infectado & 1 & 2.5 \\
$\quad$ Total & 41 & 100 \\
\hline
\end{tabular}

Se escogieron 20 casos con enfermedad pélvica inflamatoria aguda como grupo testigo, para ser tratados únicamente con Penicilina.
Cuadro 2

DISCRIMINACION DE AFECCIONES

TRATADAS SOLAMENTE CON PENICILINA

\begin{tabular}{|c|c|c|}
\hline & Casos & $\%$ \\
\hline Anexitis agudas & 12 & 60 \\
\hline Salpingitis catarral & 2 & 10 \\
\hline Abscesos tubo-ováricos & 6 & 30 \\
\hline Total & 20 & 100 \\
\hline
\end{tabular}

La pauta a seguir antes del tratamiento fue la siguiente:

\section{Cuadro 3}

ANTECEDENTES QUE SE TUVIERON EN CUENTA ANTES DEL

TRATAMIENTO TOTAL DE PACIENTES

\begin{tabular}{lc}
\hline Edad & Casos \\
\hline Menos de 20 años & 7 \\
De 20 a 30 años & 36 \\
De 30 a 40 años & 18 \\
Paridad & \\
Nulíparas & 8 \\
De 1 a 3 hijos & 26 \\
Más de 3 hijos & 27
\end{tabular}

\section{Cuadro 4}

CARACTERES CLINICOS QUE SE TUVIERON EN CUENTA ANTES DEL TRATAMIENTO. TOTAL DE PACIENTES

Temperatura

$$
\begin{aligned}
& \text { Hasta } 37^{\circ} \mathrm{C} \\
& \text { De } 37 \text { a } 38^{\circ} \mathrm{C} \\
& \text { Más de } 38^{\circ} \mathrm{C}
\end{aligned}
$$

Examen pélvico

Con tumefacción anexial

Sin tumefacción anexial

Etiología posible

Gonocóccica

Séptica o piógena

Tiempo de evolución antes del tratamiento:

Ninguna tuvo tratamiento anterior
3 pacientes

24 pacientes

34 pacientes

53 pacientes

8 pacientes

$60 \%$

$40 \%$

2 a 8 días 36 casos

8 a 12 días 25 casos 


\section{Cuadro 5}

ANALISIS DEL EXUDADO VULVOVAGINO-CERVICAL EN TODAS LAS PACIENTES ANTES DEL TRATAMIENTO

Gérmenes hallados en orden de frecuencia:

19 Gérmenes gram negativos y gram positivos sin especificación.

$2^{\circ}$ Estafilo-estreptococo

39 Colibacilo y bacilos coliformes.

49 Tricomonas, hongos y asociación de éstos.

5ㅇ Neisser.

$6{ }^{\circ}$ Proteus, Aerobacter, etc.

Cuadro 6

EXAMENES DE LABORATORIO

PRACTICADOS A TODAS LAS PACIENTES ANTES DEL

TRATAMIENTO

Fórmula roja

Resultado

\section{Leucograma}

variable

Hasta 10.000 leucocitos

Casos

Más de 10.000 leucocitos

16

45

Eritrosedimentación

Hasta 20 m.m. en la hora 3

Más de $20 \mathrm{~m} . \mathrm{m}$. en la hora

Vías de administración y dosis

La Indometacina se utilizó en todas las pacientes por vía oral en dosis de 75 mgrs. diarios, administrados en tres tomas, repartidos en $25 \mathrm{mgrs}$. con las comidas.

La Penicilina se usó parenteralmente (vía I.V., I.M.) cuya dosis, tipo y forma de administración se suministró de acuerdo con la gravedad del caso. En término medio I. M. 800.000 U. cada 12 horas. I.V. de 5.000.000 a 30 millones diarios.

Cuadro 7

EVALUACION CLINICA DESPUES DEL TRATAMIENTO

\begin{tabular}{|c|c|c|}
\hline \multicolumn{2}{|c|}{$\begin{array}{c}\text { Penicilina- } \\
\text { indometacina }\end{array}$} & \multirow{2}{*}{$\begin{array}{r}\text { Penicilina } \\
\text { Casos }\end{array}$} \\
\hline Tiempo de tratamiento & Casos & \\
\hline Hasta 10 días & 16 & 4 \\
\hline De 10 a 15 días & 12 & 2 \\
\hline De 15 a 20 días & 13 & 14 \\
\hline \multicolumn{3}{|l|}{$\begin{array}{l}\text { Cambio de tratamiento } \\
\text { por falta de }\end{array}$} \\
\hline respuesta satisfactoria & 6 & 14 \\
\hline & Casos & Caso \\
\hline \multicolumn{3}{|l|}{ Temperatura } \\
\hline Afebriles & 35 & 8 \\
\hline De 37 a $38^{\circ} \mathrm{C}$ & 4 & 10 \\
\hline Más de $38^{\circ} \mathrm{C}$ & 2 & 2 \\
\hline
\end{tabular}

En seis casos de la serie PenicilinaIndometacina, hubo necesidad de cambiar el tratamiento, por falta de res. puesta satisfactoria en la siguiente forma: dos pacientes fueron llevados a Cirugía, encontrándose en uno Embarazo ectópico roto e infectado y en el otro absceso tuvo-ovárico encapsulado, en los cuatro restantes se cambió la Penicilina por Cloramfenicol con mejoría notoria.

Debemos anotar que de las cinco pacientes con pelviperitonitis, a tres se les practicó co!potomía al ingreso.

De los 14 casos correspondientes a la serie de Penicilina sola, a dos se les practicó cirugía abdominal casi inmediata, al resto se les suministró cambio o asociación de otro antibiótico junto con el antiflogístico. (Indometacina, Fenilbutazona). De estos últimos res- 
pondieron satisfactoriamente 6 con Cloramfenicol-Indometacina, dos con Cloramfenicol-Fenilbutazona, dos con intervención quirúrgica y las otras dos se enviaron a fisioterapia en combinación con vacunas Polivalentes.

Los cambios de laboratorio se encuentran en el cuadro 8.

\section{Cuadro 8}

CAMBIOS DE LABORATORIO DESPUES DEL TRATAMIENTO

\begin{tabular}{|c|c|c|}
\hline \multicolumn{2}{|c|}{$\begin{array}{l}\text { Penicilina- } \\
\text { Indometacina }\end{array}$} & $\begin{array}{l}\text { Peni- } \\
\text { cilina }\end{array}$ \\
\hline \multicolumn{3}{|l|}{ Eritrosedimentación } \\
\hline Hasta $20 \mathrm{~m} . \mathrm{m}$. en la hora & 30 & 4 \\
\hline De 20 a $30 \mathrm{~m} . \mathrm{m}$. hora & 9 & 4 \\
\hline De 30 a $40 \mathrm{~m} . \mathrm{m}$. hora & 2 & 10 \\
\hline \multicolumn{2}{|l|}{ Más de $40 \mathrm{~m} . \mathrm{m}$. en la hora } & 2 \\
\hline \multicolumn{3}{|l|}{ Leucograma } \\
\hline Hasta 10.000 leucocitos & 32 & 5 \\
\hline $\begin{array}{l}\text { De } 10.000 \text { a } 14.000 \\
\text { leucocitos }\end{array}$ & 7 & 13 \\
\hline Más de 14.000 leucocitos & 2 & 2 \\
\hline
\end{tabular}

Cuadro 9

CONTROL DEL EXUDADO VULVOVAGINO-CERVICAL DESPUES DEL TRATAMIENTO

En orden de frecuencia

19 Gérmenes gram negativos y positivos sin especificación.

20 Bacilos coliformes y Bacilo de Doderlein.

30 Tricomonas, Hongos.

49 Proteus, seudomona auriginosa, aerobacter.

En los controles de exudado Vulvovagina-revical se puede observar la ausencia de Estafilo-Estreptococo y Neisser.

A la paciente que presentó manifestaciones cutáneas, se le suspendió el medicamento, desapareciendo éstos sin ningún otro tratamiento. A las restantes no se les suprimió la droga, regresando dichos síntomas después de 4 días de su administración.

Los resultados finales pueden apreciarse en el cuadro 10.

\section{Efectos secundarios}

\section{Cefalea moderada}

II. Náuseas y vómitos

III. Manifestaciones cutáneas

IV. Hipertrofia de ganglios regionales
3 pacientes

1 paciente

1 paciente (Habones)

1 paciente 
Cuadro 10

RESULTADOS FINALES DE LOS TRATAMIENTOS

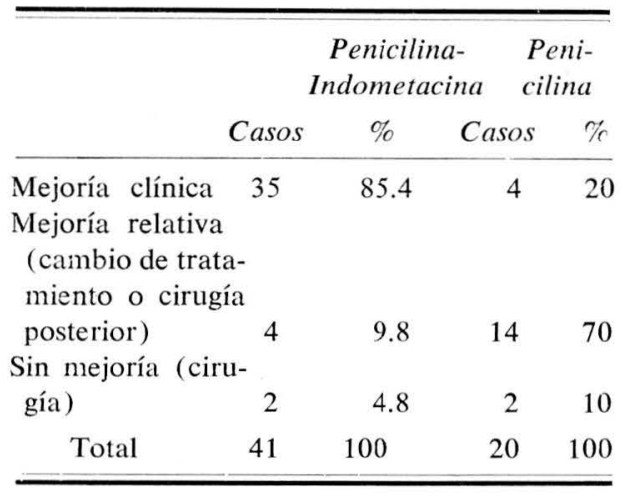

\section{Control}

Conocemos el problema de controles en las entidades hospitalarias de nuestro medio, que desofortunadamente son difíciles de llevar a cabo. Se pudo controlar el $30 \%$ de las pacientes, encontrándose hasta la fecha únicamente dos recidivas. Dichas pacien- tes tuvieron que ser llevadas a cirugía por re-agudización de las masas.

NOTA: La paciente con Hipertro fia ganglionar no acudió al control.

\section{Conclusiones}

19 La Indometacina es una droga útii en la enfermedad pélvica inflamatoria aguda.

$2^{\circ}$ Es bien tolerada y sus efectos secundarios son poco marcados en dosis de 75 miligramos diarios.

$3^{\circ} \mathrm{Su}$ administración puede prolongarse hasta por 20 días, sin apreciarse manifestaciones tóxicas dignas de tenerse en cuenta.

49 Los medicamentos antiflogísticos son indispensables como coadyuvantes en el tratamiento de la enfermedad pélvica inflamatoria aguda, en combinación con los antibióticos y demás medidas generales.

\section{BIBLIOGRAFIA}

1. AMAYA LEON HERNANDO: Comentarios a un año de internado. Tesis 1944.

2. ESPINOSA Y ESPINOSA ALVARO: Anexitis. Comentarios a tres años de estadística en el servicio de Ginecología del Hospital San Juan de Dios. Tesis, 1946.

3. AMOROCHO CARREÑO JORGE: Tratamiento de las afecciones anexoparametriales con la fenil-butazona.

4. AMOROCHO CARREÑO JORGE: Nuevas anotaciones sobre el uso de la fenil-butazona en las obstrucciones tubáricas. Rev. Col. de Obstetricia y Ginecología , II, 3, 313, 1960.

5. COLLINS CONRAD G., HANSEN FREDERIC: Treatment of the pelvic absces. Clin. Obst. and Gin., 512, Jun., 1959. 
6. CHATTAS ALBERTO: Eritrosedimentación. Su aplicación a la clínica. Salvat Editores, Buenos Aires, 1943.

7. Tratado de Ginecología Novak Jones. Sexta Edición, 1964.

8. BUTAZOLIDINA (Fenilbutazona) H. K. v. RECHENBERG (Baden Suiza ) Colaboración: H. A. KUNZ, R. DENSS, B. EXER, R. PULVER, E. G. STENGER, G. WILHELMI, 1964.

9. PAUL W. D.: Indometacina, un nuevo agente anti-inflamatorio. Arthritis and Rheumatism Foundation. Vol. 4, No 3. March, 1963.

10. SUNSHINE ABRAHAM, LASKA EUGENE, MEISNER MORRIS, MORGAN SYDNEY: "Analgesic studies of Indometacin as analized by computer techniques": Clinical Pharmacology \& Therapeutics 5 (6). Part 1.: 699-707, 1964.

\section{PREMIO CUADRIENAL DE LA FUNDACION INTERNACIONAL DE GINECOLOGIA Y OBSTETRICIA}

Valor de 10.000 francos belgas para distribuír el 199 de octubre de 1966 por la Sociedad Real Belga de Ginecología y Obstetrica al autor del mejor trabajo de Ginecología y Obstetricia, escrito o publicado en el curso de los cuatro años precedentes.

\section{B A S E S :}

1a Las trabajos presentados no pueden haber sido premiados anteriormente.

2a Deben ser enviados en los idiomas oficiales del Congreso Internacional: Alemán, Inglés, Español, Francés, Italiano.

3ạ Los trabajos deben ser enviados con dos copias al Secretario de la Sociedad Real Belga de Ginecología y Obstetrica (doctor Roger Vokaer, 309, Avenue Moliere, Bruxelles) antes del 1 o de abril de 1966.

4ạ Las decisiones del jurado serán tomadas por la mayoría de votos.

¿Qué es

$$
\text { “SEGUROS MEDICOS VOLUNTARIOS, S. A."? }
$$

Solicite informes: Apartado Aéreo No 11777 - Bogotá, D. E. 